\title{
Detección intradina en comunicaciones ópticas con modulación de fase binaria empleando un lazo de costas en el dominio del procesamiento digital de señales
}

\author{
E. A. Domínguez Retamoza ${ }^{a}$, A. Arvizu Mondragón ${ }^{a, *}$, F. Rojas Iñiguez ${ }^{b}$, F.J. Mendieta Jiménez $^{a}$, y J. Santos Aguilar ${ }^{a}$ \\ ${ }^{a}$ Centro de Investigación Científica y de Educación Superior de Ensenada, \\ Carret. Ens.-Tij. Número 3918, Zona Playitas, Ensenada, B.C., 22860, México. \\ *e-mail:arvizu@cicese.mx \\ ${ }^{b}$ Centro de Nanociencias y Nanotecnología de la UNAM, \\ Carret. Ens.-Tij. Número 3917, Colonia Playitas, Ensenada, B.C., 22860, México.
}

Received 6 October 2020; accepted 30 November 2020

\begin{abstract}
Reportamos un lazo de Costas para demodulación de un campo óptico con modulación de fase binaria (BPSK). El lazo opera en el dominio del procesamiento digital de señales (DSP) como parte de un receptor digital coherente intradino. Generalmente, la implementación práctica de demodulación BPSK en el dominio óptico no es factible. Por ello, empleamos técnicas basadas en la digitalización a alta velocidad del observable eléctrico post-fotodetección y el lazo de Costas en DSP actúa sobre la señal digitalizada. Elegimos este lazo por ser una estructura óptima tanto de demodulación BPSK como de sincronización de portadora. Normalmente, los lazos de Costas para demodulación BPSK operan con una portadora con frecuencia mayor que la velocidad del bit. Sin embargo, como demostramos en este trabajo, este lazo también puede operar adecuadamente para una velocidad de bit mayor que la frecuencia de portadora (detección intradina). A lo mejor de nuestro conocimiento, el lazo de Costas en DSP para demodulación BPSK intradina se reporta por primera vez en este trabajo. Para el diseño del lazo de Costas intradino nos basamos en el modelo PLL equivalente de un lazo de Costas convencional. Con fines de comparación y evaluación del desempeño, primero implementamos en simulación, un lazo de Costas tradicional y posteriormente, uno intradino. Para nuestras simulaciones, empleamos señales del mundo real (datos binarios y ruido aditivo) adquiridas mediante digitalización. Finalmente, se realizó la caracterización del lazo de Costas intradino empleando una señal eléctrica post-fotodetección digitalizada obtenida a la salida de nuestro receptor digital coherente. Se observó un desempeño muy cercano al obtenido mediante simulación.
\end{abstract}

Descriptores: Detección óptica coherente; lazo de costas; detección intradina.

We report a Costas loop for demodulation of an optical field with binary phase modulation (BPSK). The loop operates in the domain of digital signal processing (DSP) as part of an intradyne coherent digital receiver. The practical implementation of BPSK demodulation in the optical domain is generally not feasible. For this reason, we use techniques based on high-speed digitization of the post-photodetection electrical observable, and the Costas loop in DSP acts on the digitized signal. We chose this loop because it is an optimal structure for both BPSK demodulation and carrier synchronization. Typically, Costas loops for BPSK demodulation operate on a carrier with a frequency higher than the bit rate. However, as we demonstrate in this work, this loop can also perform adequately for a bit rate higher than the carrier frequency (intradyne detection). To the best of our knowledge, the Costas loop in DSP for intradyne BPSK demodulation is reported for the first time in this work. To design the intradyne Costas loop, we used the PLL-equivalent model of a conventional Costas loop. For comparison and performance evaluation, we implemented in simulation, first, a traditional Costas loop and later an intradyne one. We use real-world signals (binary data and additive noise) acquired by digitization for our simulations. Finally, we performed the intradyne Costas loop's characterization using a digitized post-photodetection electrical signal obtained at the output of our digital coherent receiver. We observe a performance very close to that obtained by simulation.

Keywords: Optical coherent detection; costas loop; intradyne detection.

PACS: 42.79.Sz

DOI: https://doi.org/10.31349/RevMexFis.67.238

\section{Introducción}

La transmisión de información empleando fuentes ópticas a través del canal óptico atmosférico o fibrado ha ido evolucionando desde los denominados sistemas de modulación de intensidad y detección directa (Fig. 1), que incluyen aplicaciones comerciales, tales como SONET y radio sobre fibra óptica [1], hasta los sistemas de modulación y detección más complejos, tales como los denominados sistemas con detección óptica coherente (Fig. 2a), donde se puede aprovechar el bagaje desarrollado en el dominio de las radiofrecuencias para modulaciones multinivel [1].
Durante los años 80 y parte de los 90 del siglo pasado, se trabajó extensivamente en la investigación teórica y experimental enfocada en la implementación práctica de sistemas de detección óptica coherente (heterodina y homodina, ver Fig. 2a). Sin embargo, se encontró que debido principalmente a las limitaciones impuestas por el ruido de fase inherente de las fuentes ópticas (láseres de semiconductor) [2], su implementación práctica en el dominio óptico no era factible. Esto ocasionó que el interés por los mismos fuera decayendo significativamente, pero gracias a un importante avance en el desarrollo de sistemas de digitalización y procesamiento digital 
de señales de muy alta velocidad, fue posible trasladar parte de la detección óptica coherente al dominio del procesamiento digital de señales [2,3]. Esto permitió la implementación práctica de los sistemas de comunicaciones ópticas coherentes con la ventaja adicional de poder compensar algunas de las perturbaciones introducidas por el canal de comunicaciones [2,3], ver Fig. 2b. Así, a pesar de que actualmente ya existen comercialmente algunos sistemas de comunicación óptica coherente (intradinos), la investigación teórica y práctica de sistemas de modulación multinivel de intensidad, fase y/o estado de polarización de la luz con recepción óptica coherente se mantiene activa [4-6].

Generalmente, los sistemas de comunicaciones ópticas comerciales emplean como fuentes ópticas láseres de semiconductor operando en un régimen "clásico", es decir, con un número relativamente alto de fotones por bit en la etapa de transmisión. Así los sistemas más comunes son los de modulación de intensidad y detección directa (ver Fig. 1). En ellos, para recuperar la información enviada a través de una portadora óptica se requiere solamente del uso de un fotodetector, y por eso se le denomina detección directa. El fotodetector realiza la doble función de convertir la señal óptica a eléctrica y demodular la información presente en la portadora óptica. Sin embargo, al realizar la detección directa, no se preserva la información que podría presentarse en la fase, la frecuencia y/o el estado de polarización del campo óptico. Es en estos casos cuando debe emplearse la denominada detección óptica coherente, que puede ser heterodina, homodina o intradina (ver Fig. 2). La detección óptica coherente requiere el uso de un oscilador local óptico (un láser) en la etapa de recepción, lo cual agrega mayor complejidad que la detección; directa,sin embargo, la detección coherente tiene la ventaja de una amplificación coherente gracias al uso de dicho oscilador óptico [2]. No obstante lo anterior para este proceso de fotodetección coherente se requiere que el oscilador local óptico tenga una relación de fase constante (sincronía de fase) con respecto al campo óptico incidente. Este problema específico implica que desde un punto de vista práctico, no es factible realizar el proceso en el dominio óptico con componentes comerciales [2,3].

Por esta razón, se propuso una técnica alternativa, la detección óptica intradina,ver Fig. 3, donde se utiliza un oscilador local óptico operando libremente (free running), sin

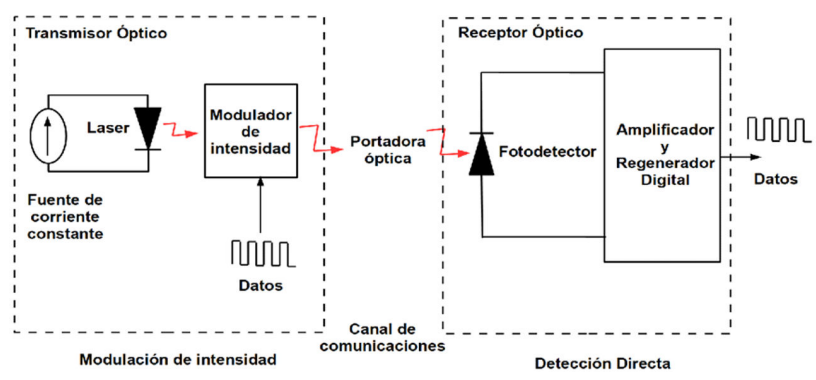

FIgURA 1. Diagrama a bloques de un sistema típico de comunicaciones ópticas con modulación de intensidad y detección directa operando con datos en banda base.

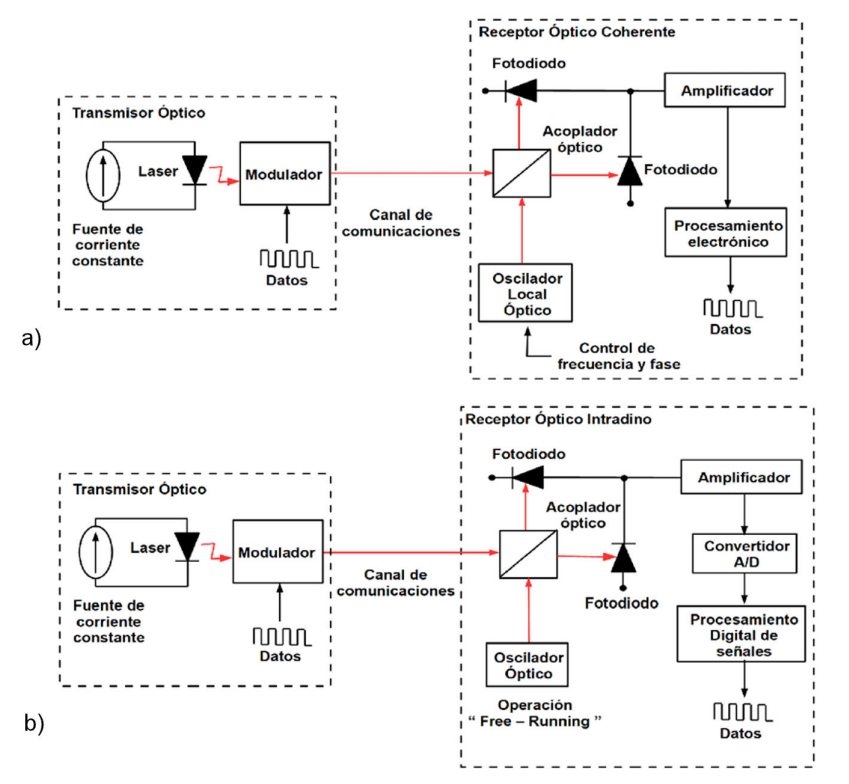

FIGURA 2. Sistema de comunicaciones ópticas con detección coherente. a) Heterodino $\left(\left|f_{c}-f_{o}\right|>B_{r}\right)$ o homodino $\left(f_{c}-f_{o}=0\right)$ : dominio óptico, b) intradino: dominio del procesamiento digital de señales $\left(0<\left|f_{c}-f_{o}\right|<B_{r}\right)$. Donde: $f_{c}, f_{o}$ : frecuencias de la portadora y oscilador local respectivamente, $B_{r}$ : velocidad de bit y $\left|f_{c}-f_{o}\right|:$ señal de batimiento. A/D: analógico a digital.

una relación de fase constante con respecto a la señal óptica de información, aunque sí debe estabilizarse en longitud de onda (para que la señal de batimiento esté dentro de la banda de frecuencias del fotodetector). En este caso, proceso de sincronía de fase se realiza en el dominio del procesamiento digital de señale y actúa sobre el observable óptico postfotodetección digitalizado (ver Fig. 2).

Debido a la relevancia de los sistemas intradinos para la transmisión de información de ultra alta velocidad (decenas o centenas de Gigabits por segundo), ha habido una actividad importante de investigación y desarrollo de diversos algoritmos para la estimación de fase óptica y compensación de perturbaciones para sistemas de comunicaciones ópticas "clasicos". En estos es prioritario realizar el procesamiento en tiempo real a muy alta velocidad; por ello, se privilegia el uso de algoritmos eficientes [2,3]. Por otro lado, en aplicaciones de más baja velocidad, por ejemplo, en criptografía cuántica, o en comunicaciones ópticas satelitales, no se tiene esa restricción, pues las velocidades típicamente empleadas oscilan alrededor de algunas decenas de Megabits por segundo, lo que permite hacer uso de otros algoritmos que puedan resultar óptimos desde el punto de vista de la teoría estadística de las comunicaciones [7-9].

Así, el detector óptico intradino motivo del presente trabajo se desarrolló teniendo en mente aplicaciones para criptografía cuántica y/o comunicación óptica satelital y utilizando modulación BPSK del campo óptico. Con base en las consideraciones anteriores, implementamos un receptor digital coherente intradino para espacio libre (ver Figs. 3 y 8 ) y seleccionamos un estimador de fase óptimo y el lazo de Costas 


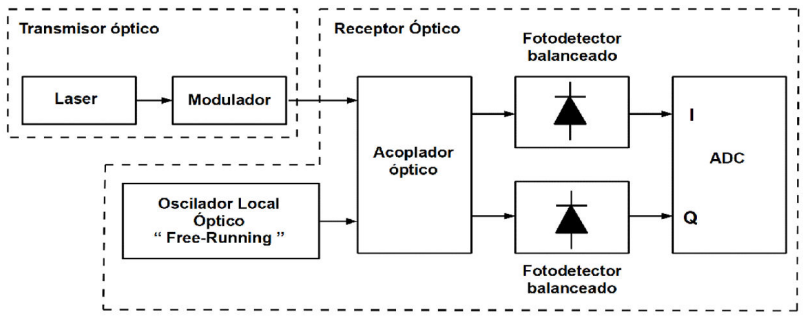

FIGURA 3. Sistema de comunicaciones ópticas con receptor digital coherente intradino, donde el oscilador local opera en modo freerunning. ADC: convertidor analógico a digital. El tipo de acoplador óptico utilizado es un dispositivo denominado híbrido óptico, el cual está diseñado para extraer las cuadraturas, es decir, los componentes real $(I)$ e imaginario $(Q)$ del campo óptico complejo resultante de la mezcla del láser de información y del oscilador local óptico.

[7-13], implementado en el dominio del procesamiento digital de señales y actuando sobre el observable eléctrico postfotodetección digitalizado.

El lazo de Costas se emplea cuando, de facto, la portadora tiene una frecuencia mayor a la velocidad del bit. Sin embargo, como demostramos en este trabajo, este lazo también puede utilizarse cuando la velocidad del bit es mayor que la frecuencia de portadora (caso intradino); hasta donde sabemos esta es la primera vez que se reporta su funcionamiento bajo estas condiciones. A continuación, describiremos algunos conceptos básicos relacionados con el funcionamiento de un lazo de Costas.

\section{Demodulación de señales BPSK ópticas}

Para recuperar la información transmitida empleando la modulación binaria de fase óptica (BPSK) se requiere de una etapa de sincronización de la portadora óptica en el receptor. Los subsistemas comúnmente empleados para llevar a cabo esta operación son el lazo de amarre de fase (PLL: phase lock loop, por sus siglas en inglés), cuando existe portadora residual, o el lazo de Costas, cuando se transmite BPSK con portadora suprimida, como es el caso del presente trabajo [14]. Dado que la operación y el diseño del lazo de Costas se puede explicar empleando un modelo más simple basado en el PLL, primero describiremos de manera general la operación de este lazo [15-18].

\subsection{Lazo de amarre de fase (PLL)}

Los lazos de amarre de fase, ver Fig. 4a), son dispositivos que han sido estudiados exhaustivamente desde las primeras décadas del siglo pasado y siguen siendo motivo de una actividad intensa de investigación y desarrollo tecnológico por tener una amplia variedad de aplicaciones en áreas tan diversas como la electrónica de consumo, el control, la metrología y las telecomunicaciones. Naturalmente, el conocimiento sobre sus principios de operación y la forma de implementarlo han evolucionado simultáneamente con la tecnología electrónica, el software y más recientemente, con la fotónica [16].
Es conveniente mencionar que el propósito principal de un PLL es "encadenar" la frecuencia de la señal $V_{y}(t)$ de un oscilador local (VCO) con una señal de referencia $V_{x}(t)$. Se entiende por encadenamiento el momento cuando el oscilador local sigue las fluctuaciones instantáneas de la fase de la señal de referencia. Para describir su funcionamiento, nos basaremos en la Fig. 4 a y en las Ecs. (1)-(3).

Al usar un detector de fase (en este caso, un multiplicador), se comparan las fases de las señales $V_{x}(t)$ y $V_{y}(t)$. A la salida del detector se obtiene la señal $v_{m}(t)$, que contiene entre otros términos- uno que es proporcional a la diferencia de fase entre $V_{x}(t)$ y $V_{y}(t)$ (segundo término de la Ec. (3)). Es pertinente mencionar que la teoría del PLL fue desarrollada considerando que se trabajaba con señales eléctricas analógicas, por eso, para obtener una señal proporcional a la diferencia de fase de dos señales, puede emplearse un multiplicador, como se muestra a continuación. Aunque el uso de dicha teoría puede aplicarse también cuando las señales $V_{x}(t)$ y $V_{y}(t)$ son ópticas y considerando que no existe un dispositivo físico que lleve a cabo la operación de multiplicación en el dominio óptico, deben buscarse alternativas para obtener la señal de diferencia de fase (ver, por ejemplo, la Ref. [13]).

$$
\begin{aligned}
V_{x}(t) & =A \operatorname{sen}\left(2 \pi f_{i} t+\theta_{i}[t]\right) \\
V_{y}(t) & =A \cos \left(2 \pi f_{0} t+\theta_{0}[t]\right) \\
v_{m}(t) & =\frac{A B}{2}\left(\operatorname{sen}\left[2 \pi\left\{f_{i}+f_{0}\right\} t+\theta_{i}\{t\}+\theta_{0}\{t\}\right]\right. \\
& \left.+\operatorname{sen}\left[2 \pi\left\{f_{i}+f_{0}\right\} t+\theta_{i}\{t\}+\theta_{0}\{t\}\right]\right),
\end{aligned}
$$

donde $A, B, f_{i}, f_{0}, \theta_{i}(t), \theta_{0}(t)$ son las amplitudes, frecuencias y fases de $V_{x}(t)$ y $V_{y}(t)$, respectivamente. La señal de diferencia de fase $v_{m}(t)$ entra al filtro de lazo (filtro pasobajas con respuesta al impulso $f(t)$ y función de transferencia $F(s)$ ), el cual determina la respuesta dinámica del sistema y elimina los términos de alta frecuencia (en este caso, el término de la Ec. (3) que contiene $\left(f_{i}+f_{0}\right)$ ), produciendo una señal de voltaje $\left(v_{c}(t)\right)$ que controla al VCO de una forma tal que su fase instántanea se ajuste para reducir (o en su defecto, mantener constante) la diferencia de fase entre la señal de entrada y el oscilador local (en este caso se dice que el PLL está “encadenado" o "amarrado”).

$$
\begin{aligned}
v_{c}(t) & =v_{m}(t) * f(t)=\frac{A B}{2} \\
& \times \operatorname{sen}\left(2 \pi\left[f_{i}-f_{0}\right] t+\theta_{i}[t]-\theta_{0}[t]\right),
\end{aligned}
$$
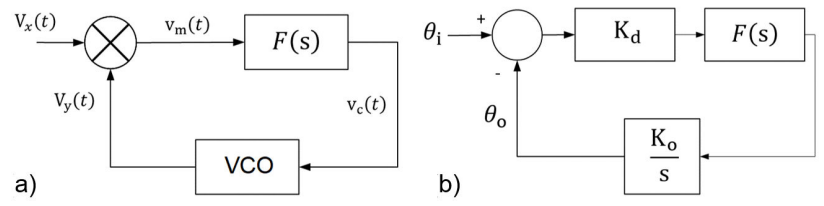

FIgURA 4. a) Diagrama a bloques de un PLL, b) diagrama de un PLL linealizado. 
donde la operación * es la convolución. Cuando el PLL está encadenado $f_{i}=f_{0}$ y la Ec. (4) se reduce a

$$
v_{c}(t)=\frac{A B}{2} \operatorname{sen}\left(\theta_{i}[t]-\theta_{0}[t]\right),
$$

esta se acostumbra linealizar suponiendo que el error de fase es pequeño, es decir, $\left(\theta_{i}[t]-\theta_{0}[t]\right) \rightarrow 0$ y dado que la función $\operatorname{sen}($.$) para un argumento pequeño se puede aproximar$ al $\operatorname{argumento} \operatorname{sen}(z) \approx z$, se tiene:

$$
v_{c}(t) \approx K_{d}\left(\theta_{i}[t]-\theta_{0}[t]\right),
$$

con

$$
K_{d}=\frac{A B}{2} \frac{V}{\mathrm{rad}} .
$$

La señal de control $v_{c}(t)$ entra al VCO, el cual variará su fase instantánea (frecuencia) en función de dicho voltaje para seguir las variaciones que se presenten en el error de fase instantáneo. A partir de las Ecs. (4) y (6) puede obtenerse un modelo linealizado del PLL, tal como se ilustra en la Fig. 4b [18]. El comportamiento de un PLL no encadenado puede obtenerse mediante el uso de herramientas gráficas, tales como los planos de fase [15] o mediante el uso de una ecuación integrodiferencial estocástica (la ecuación de Fokker-Planck) [16]. Es importante mencionar que para que el PLL se amarre y permanezca en dicha condición, debe tenerse especial cuidado en la elección del VCO, del detector de fase a utilizar y del diseño del filtro de lazo acorde a la respuesta dinámica deseada $[15,16,18]$.

\subsubsection{Lazo de Costas}

En el inciso anterior se describió el funcionamiento de un PLL, el cual, si se utiliza para la recepción de señales BPSK, requiere de la presencia de un componente de portadora residual (señal de referencia) al cual encadenarse. Esto es factible solamente con la denominada modulación BPSK imperfecta. La modulación BPSK perfecta, sin portadora residual, es la técnica de modulación de fase binaria más eficiente, pues no hay ningún desperdicio de potencia [15], pero para su demodulación requiere del uso de algún otro tipo de lazo, tal como el Lazo de Costas que describiremos a continuación.

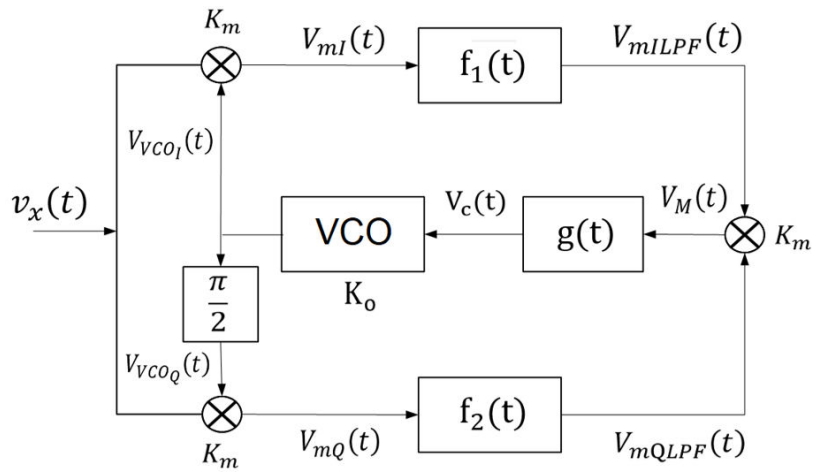

Figura 5. Diagrama a bloques de un lazo de Costas.
En el Lazo de Costas (Fig. 5), la fase de la portadora es extraída a partir de una señal BPSK de portadora suprimida $v_{x}(t)$, multiplicando los voltajes de entrada a los detectores de fase (multiplicadores) con la señal $V_{V C O_{I}}(t)$ producida a la salida del VCO y con una versión con 90 grados de desfasamiento de la misma, $V_{V C O_{Q}}(t)$, filtrando los resultados y multiplicándolos para que, a partir del resultado filtrado, se obtenga una señal para controlar la fase y frecuencia del VCO del lazo.

La señal BPSK de portadora suprimida $v_{x}(t)$ se puede expresar como [19]:

$$
v_{x}(t)=d(t) \operatorname{sen}\left(2 \pi f_{i} t+\theta_{i}[t]\right),
$$

donde $d(t)$ es la señal de datos con velocidad de bit $B_{r}$ (bits por segundo) y:

$$
d(t)=\left\{\begin{array}{c}
\text { 1_para _"1" } 1 \text { _lógico } \\
\text {-1_para_" }{ }^{\prime \prime} \text { lógico }
\end{array} .\right.
$$

El oscilador controlado por voltaje entrega una señal $V_{V C O_{I}}(t)$, la cual -cuando se desfasa 90 grados- produce la señal $V_{V C O_{Q}}(t)$ de acuerdo con las ecuaciones siguientes:

$$
\begin{aligned}
V_{V C O_{I}}(t) & =C \operatorname{sen}\left(2 \pi f_{0} t+\theta_{0}[t]\right), \\
V_{V C O_{Q}}(t) & =C \cos \left(2 \pi f_{0} t+\theta_{0}[t]\right) .
\end{aligned}
$$

donde $f_{i}, f_{0}, \theta_{i}(t), \theta_{0}(t)$ son las frecuencias y fases de la portadora y del oscilador local (VCO), respectivamente, y $C$ es la amplitud de ambas. Denotamos las salidas de los multiplicadores (con ganancia $K_{m}$ ) de las ramas superior e inferior del lazo como $V_{m I}(t)$ y $V_{m Q}(t)$, respectivamente (Fig. 5):

$$
\begin{aligned}
V_{m I}(t) & =K_{m} d(t) \operatorname{sen}\left(2 \pi f_{i} t+\theta_{i}[t]\right) C \operatorname{sen}\left(2 \pi f_{0} t+\theta_{0}[t]\right) \\
& =\frac{K_{m} C d(t)}{2}\left(\cos \left[2 \pi\left\{f_{i}-f_{0}\right\} t+\theta_{i}\{t\}-\theta_{0}\{t\}\right]\right. \\
& \left.-\cos \left[2 \pi\left\{f_{i}+f_{0}\right\} t+\theta_{i}\{t\}+\theta_{0}\{t\}\right]\right) \\
V_{m Q}(t) & =K_{m} d(t) \operatorname{sen}\left(2 \pi f_{i} t+\theta_{i}[t]\right) C \cos \left(2 \pi f_{0} t+\theta_{0}[t]\right) \\
& =\frac{K_{m} C d(t)}{2}\left(\operatorname{sen}\left[2 \pi\left\{f_{i}-f_{0}\right\} t+\theta_{i}\{t\}-\theta_{0}\{t\}\right]\right. \\
& \left.+\operatorname{sen}\left[2 \pi\left\{f_{i}+f_{0}\right\} t+\theta_{i}\{t\}+\theta_{0}\{t\}\right]\right)
\end{aligned}
$$

Normalmente, el ancho de banda de los filtros paso-bajas (LPF) de las ramas $f_{1}(t)$ y $f_{2}(t)$ se escoge de tal forma que elimine los términos de sumas de frecuencia de las Ecs. (11) y (12) [20]. Entonces la salida de los filtros viene dada por las señales:

$$
\begin{aligned}
V_{m I L P F}(t) & =\frac{K_{m} C d(t)}{2} \\
& \times\left(\cos \left[2 \pi\left\{f_{i}-f_{o}\right\} t+\theta_{i}\{t\}-\theta_{0}\{t\}\right]\right), \\
V_{m Q L P F}(t) & =\frac{K_{m} C d(t)}{2} \\
& \times\left(\operatorname{sen}\left[2 \pi\left\{f_{i}-f_{o}\right\} t+\theta_{i}\{t\}-\theta_{0}\{t\}\right]\right),
\end{aligned}
$$


y la multiplicación de ambas es:

$$
\begin{aligned}
V_{M}(t) & =V_{m I L P F}(t) V_{m Q L P F}(t), \\
V_{M}(t) & =\frac{\left(K_{m} C d[t]\right)^{2}}{4} \\
& \times\left(\cos \left[2 \pi\left\{f_{i}-f_{o}\right\} t+\theta_{i}\{t\}-\theta_{0}\{t\}\right]\right) \\
& \times\left(\operatorname{sen}\left[2 \pi\left\{f_{i}-f_{o}\right\} t+\theta_{i}\{t\}-\theta_{0}\{t\}\right]\right),
\end{aligned}
$$

cuando $f_{i}=f_{0}$, se dice que el lazo está encadenado y definiendo el error de fase $\theta_{e}(t)=\theta_{i}(t)-\theta_{0}(t)$, las ecuaciones anteriores se reducen a:

$$
\begin{aligned}
V_{m I L P F}(t) & =\frac{K_{m} C d(t)}{2}\left(\cos \left[\theta_{e}\{t\}\right]\right) \\
V_{m Q L P F}(t) & =\frac{K_{m} C d(t)}{2}\left(\operatorname{sen}\left[\theta_{e}\{t\}\right]\right), \\
V_{M}(t) & =\frac{\left(K_{m} C d[t]\right)^{2}}{4}\left(\cos \left[\theta_{e}\{t\}\right]\right)\left(\operatorname{sen}\left[\theta_{e}\{t\}\right]\right) \\
& =\frac{\left(K_{m} C d[t]\right)^{2}}{4} \frac{1}{2} \operatorname{sen}\left(2 \theta_{e}[t]\right),
\end{aligned}
$$

si el error de fase es pequeño $\operatorname{sen}\left(\theta_{e}(t)\right) \approx \theta_{e}(t)$ y dado que $d(t)^{2}=1$ pues $d(t)= \pm 1$, entonces:

$$
V_{M}(t)=\frac{\left(K_{m} C\right)^{2}}{4} \theta_{e}(t)=K_{d}\left(\theta_{i}[t]-\theta_{0}[t]\right)
$$

con $K_{d}=\left(K_{m} C\right)^{2} / 4$. A esta constante se le denomina ganancia del detector de fase equivalente y sus unidades son [Volts/radian]. Los filtros de las ramas superior e inferior del lazo se definen de acuerdo con las siguientes funciones de transferencia [21,22]:

$$
\begin{aligned}
F_{1}(s) & =\mathcal{L}\left\{f_{1}(t)\right\}=F_{2}(s)=\mathcal{L}\left\{f_{2}(t)\right\}=\mathcal{L}\{f(t)\} \\
& =F(s)=\frac{1}{1+s \tau} \\
G(s) & =\mathcal{L}\{g(t)\}=\frac{1+s \tau_{2}}{s \tau_{1}},
\end{aligned}
$$

donde $\mathcal{L}$ es la transformada de Laplace y las constantes de tiempo $\tau, \tau_{1}$ y $\tau_{2}$ sirven para determinar el comportamiento

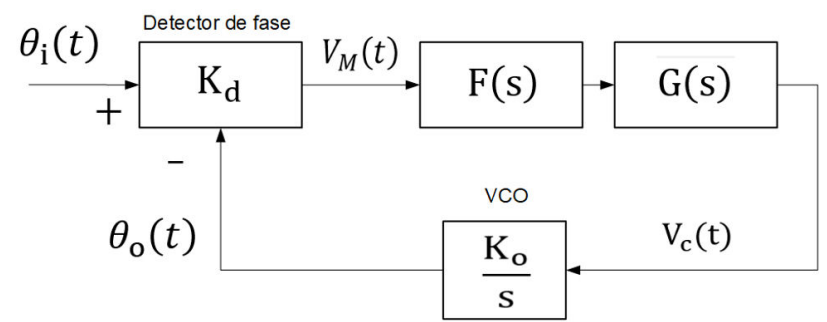

Figura 6. Modelo PLL equivalente de un lazo de Costas.

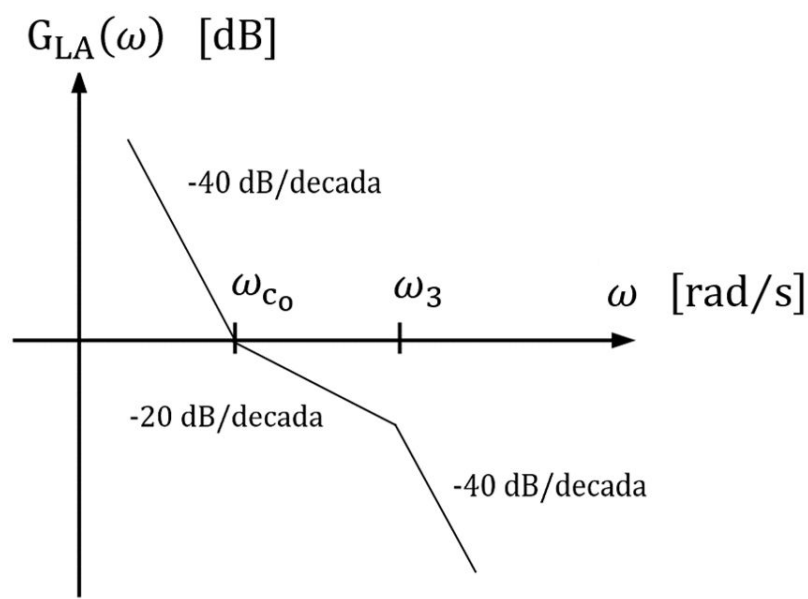

FIguRA 7. Diagrama de Bode de la ganancia de lazo abierto del modelo PLL equivalente de un lazo de Costas.

dinámico del lazo; más adelante se describe cómo se seleccionan sus valores. Con base en las Ecs. (19) a (21) del lazo de Costas encadenado, se plantea un modelo similar al de un PLL linealizado [18], (ver Figs. 4b y 6), donde se observa un detector de fase equivalente con ganancia $K_{d}$ de acuerdo con la Ec. (19) y el VCO se modela como un integrador [18] con ganancia $K_{0}[\mathrm{rad} / \mathrm{s} /$ Volt]. Los filtros $F(s)$ y $G(s)$ están definidos por las Ecs. (20) y (21), respectivamente. Definimos la ganancia de lazo abierto del PLL equivalente $G_{L A}(s)$ como:

$$
G_{L A}(s)=\frac{k_{0}}{s} K_{d} \frac{1}{1+s \tau} \frac{1+s \tau_{2}}{s \tau_{1}} .
$$

Para fines de diseño se obtiene el diagrama de Bode de $G_{L A}(s)$ [18], ver Fig. 7, donde las pendientes de las asíntotas están determinadas por los 3 polos y el cero de la función de transferencia. A la frecuencia angular de cruce por cero decibeles se le denotará como [18]:

$$
\omega_{c o}=\frac{1}{\tau_{2}}
$$

además,

$$
\omega_{3}=\frac{1}{\tau} .
$$

El lazo de Costas es un dispositivo único pues realiza tanto la reconstrucción de la portadora (suprimida) como la detección de datos dentro del mismo lazo [20]. La rama superior del lazo es llamada rama en fase $(I)$ o de sincronización y funciona como un PLL típico entregando una señal de error "corrupta" $V_{M}(t)$. La rama inferior, en cuadratura $(Q)$ o de decisión, provee la extracción de los datos y corrige la "corrupción" de $V_{M}(t)$. La señal de error corregida, se aplica a través del filtro de lazo $G(s)$ al VCO, el cual tiene un estimado de fase de la portadora (suprimida).

\subsection{Diseño de un lazo de costas}

El diseño de un lazo de Costas consiste en determinar los valores de las diversas ganancias del lazo y de las constantes 
de tiempo de los filtros paso-bajas utilizados. En esta sección se describe la metodología empleada para diseñar lazos de Costas como demoduladores de BPSK perfecta con base en las ecuaciones desarrolladas en la sección anterior. El modelo PLL equivalente del lazo de Costas y el diagrama de Bode para la Ganancia de lazo abierto $G_{L A}(s)$ de dicho lazo. Nuestro propósito es utilizar un lazo de Costas en DSP operando para el caso intradino, pero por conveniencia y para fines de comparación de desempeño, diseñaremos en primer lugar un lazo de Costas "convencional" $\left(f_{c}>B_{r}\right)$ y más adelante diseñaremos uno para el caso intradino $\left(f_{c}<B_{r}\right)$.

Así, para el lazo de Costas "convencional" elegiremos como frecuencia portadora $f_{c}=200 \mathrm{MHz}$ y una velocidad de bit $\mathrm{Br}=50 \mathrm{Mbps}$. Proponemos las ganancias de los multiplicadores $K_{m}=2$ y con $C=1$, entonces $K_{d}=$ $\left(K_{m} C\right)^{2} / 4=1$ Volts/rad. Dado que estamos diseñando un sistema retroalimentado, es importante mantener un margen de seguridad con base en un criterio de estabilidad, en particular, usando el diagrama de Bode del lazo (Fig. 9) [15,18]. Así, se define una frecuencia angular $\omega_{T}$, tal que:

$$
0.05 \omega_{c} \leq \omega_{T} \leq 0.1 \omega_{c},
$$

con $\omega_{c}=2 \pi f_{c}$, donde $f_{c}=200 \mathrm{MHz}$, es la frecuencia de la portadora. Esto asegura que para $\left|G_{L A}(\omega)\right|_{d B}=0$, $\arctan \left(G_{L A}(\omega)\right) \neq 180^{\circ}$ (criterio de estabilidad) $[15,18]$. Para nuestro diseño seleccionamos $\omega_{T}=0.1 \omega_{c}=$ $0.1(2 \pi) 200\left(10^{6}\right)=1.2566 \times 10^{8} \mathrm{rad} / \mathrm{s}$, y al observar la Fig. 7 decidimos escoger:

$$
\omega_{c o}=\omega_{T}=\frac{1}{\tau_{2}}=1.2566 \times 10^{8} \frac{\mathrm{rad}}{\mathrm{s}},
$$

con este valor se obtiene:

$$
\tau_{2}=\frac{1}{\omega_{c o}}=7.9577 \times 10^{-9} \mathrm{~s} .
$$

También de la Fig. 7 se observa que $\left|G_{L A}(\omega)\right|_{(\omega=}=$ $\left.\omega_{c} O\right)=0 \mathrm{~dB}$ lo cual corresponde en una escala lineal a

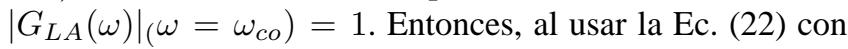
$s=j \omega$, se obtiene:

$$
G_{L A}(j \omega)=-K d \frac{K_{0}}{\omega^{2} \tau_{1}}\left(\frac{1+j \omega\left[\tau_{2}+\tau\right]+\omega^{2} \tau_{2} \tau}{1+\omega^{2} \tau^{2}}\right) .
$$

Para el diseño de los filtros con función de transferencia $F(s)$ Ec. (20), debe considerarse la conveniencia de tener una frecuencia de corte $\left(\omega_{3}=2 \pi f_{3}\right)$ suficientemente grande para recuperar fielmente la señal de datos [20]. Por otro lado, estos filtros deben suprimir los términos del doble de frecuencia de portadora, en este caso $2 f_{c}=2 \times 200 \mathrm{MHz}=400 \mathrm{MHz}$; así, seleccionamos:

$$
\begin{aligned}
\omega_{3} & =2 \pi f_{3}=2 \pi \times 2 B_{r} 2 \pi \times 2 \times 50 \times 10^{6} \frac{\mathrm{rad}}{\mathrm{s}} \\
& =6.2832 \times 10^{8} \frac{\mathrm{rad}}{\mathrm{s}},
\end{aligned}
$$

entonces, de la Ec. (24):

$$
\tau=\frac{1}{\omega_{s}}=1.5915 \times 10^{-9} \mathrm{~s} .
$$

Al sustituir (29) y (26) en (27) y calcular su magnitud, se obtiene:

$$
\left|G_{L A}(j \omega)\right|=K_{d} \frac{K_{0}}{\omega^{2} \tau_{1}} \sqrt{\left(\frac{1+\left[\omega^{2}\left\{1.2665 \times 10^{-17}\right\}\right]^{2}+\left[\omega\left\{6.3662 \times 10^{-9}\right\}\right]^{2}}{1+\left[\omega^{2}\left\{2.5329 \times 10^{-8}\right\}\right]^{2}}\right)} .
$$

Los términos de $\omega$ dentro del radical resultan despreciables con respecto al valor de 1 al ser evaluados para frecuencias menores o iguales a la frecuencia de portadora, es decir, para $\omega=2 \pi \times 200 \times 10^{6} \mathrm{rads} / \mathrm{s}$, que es el intervalo de frecuencias en que se espera que trabaje el lazo, así:

$$
\left|G_{L A}(j \omega)\right| \approx K_{d} \frac{K_{0}}{\omega^{2} \tau_{1}}
$$

para

$$
\omega \leq 2 \pi \times 200 \times 10^{6} \frac{\mathrm{rad}}{\mathrm{s}},
$$

y al recordar que $\left|G_{L A}(j \omega)\right|_{\omega=\omega_{c 0}}=1$,

$$
\left|G_{L A}(j \omega)\right|_{\omega=\omega_{c 0}}=1=K_{d} \frac{K_{0}}{\omega_{c o}^{2} \tau_{1}}=\frac{K_{0}}{\omega_{c o}^{2} \tau_{1}},
$$

entonces:

$$
\omega_{c o}^{2}=\frac{K_{0}}{\tau_{1}}
$$

Por otro lado, para un PLL de segundo orden se tiene (considerando el modelo PLL equivalente del lazo de Costas, Fig. 6) $[15,18]$ :

$$
\omega_{n}=\sqrt{\frac{K_{d} K_{0}}{\tau_{1}}}
$$

donde $\omega_{n}$ es la frecuencia angular natural del sistema y como $K_{d}=1$ :

$$
\omega_{n}^{2}=\frac{K_{0}}{\tau_{1}}=\omega_{c 0}^{2}=1.2566 \times 10^{8} \frac{\mathrm{rad}}{\mathrm{s}} .
$$

También para un PLL de segundo orden se tiene que el factor de amortiguamiento $\zeta$ viene dado por $[15,18]$ :

$$
\zeta=\frac{\omega_{n} \tau_{2}}{2}
$$

y dado que $\tau_{2}=1 / \omega_{c o}=1 / \omega_{n} \Rightarrow \zeta=0.5$ y para determinar el valor de $\tau=1$ usamos (31): 


$$
\omega_{c o}^{2}=\frac{K_{0}}{\tau_{1}}=1.5790 \times 10^{16} .
$$

De donde $\tau_{1}=6.3329 \times 10^{-17} K_{o}$. Como en esta ecuación se tienen dos incógnitas, propondremos una de ellas para encontrar la otra; así, por ejemplo, si elegimos la ganancia del VCO como $20 \mathrm{MHz} /$ Volt, es decir $K_{o}=2 \pi \times 20 \times 10^{6}$ $(\mathrm{rads} / \mathrm{s}) / \mathrm{volt}$, entonces:

$$
\tau_{1}=7.9582 \times 10^{-9} \mathrm{~s} .
$$

Así, con los parámetros obtenidos de este diseño (ver Tabla I), puede implementarse el lazo de Costas de la Fig. 5.

Para el diseño del lazo de Costas Intradino procedemos de forma similar, pero ahora utilizaremos una frecuencia de portadora $f_{c}=30 \mathrm{MHz}$ (la elección de este valor se explica en la siguiente sección). En este caso, los parámetros obtenidos son los indicados en la Tabla II.

\section{Implementación y caracterización de un Lazo de Costas en DSP}

El lazo de Costas Intradino que diseñamos en este trabajo es parte de un receptor digital coherente (RDC) para comunicaciones ópticas con modulación BPSK perfecta. El RDC está pensado para aplicación en comunicaciones ópticas satelitales. En la Fig. 8 mostramos el RDC que implementamos. La fuente óptica que se emplea en la etapa de transmisión (no incluida en la Fig. 8) es un láser Teraxion Purespectrum NLL operando a una longitud de onda de $1550 \mathrm{~nm}$. Su salida entra al modulador de fase óptica IXBlue Modelo MPZ-LN20-FA-FA. Este modulador es manejado por una secuencia pseudoaletoria eléctrica de datos con longitud PRBS-6 y velocidad de bit $B_{r}=50 \mathrm{Mbps}$, generada por el Analizador Digital de Transmisión HP3784A. El modulador de la señal óptica de datos es un modulador de fase fabricado con cristales de Niobato de Litio. Estos cristales electro-ópticos son sensibles a la polarización, por tanto, la señal óptica debe llegar correctamente polarizada al cristal. Para esto se utilizan fibras birrefringentes que mantienen la polarización y un láser que emite una señal óptica polarizada y alineada a la fibra óptica. Se utilizaron diferentes distancias para las pruebas de transmisión en espacio libre; las mediciones que se reportan alcanzan $500 \mathrm{~m}$ con cielo despejado. El campo óptico a la salida del modulador es inyectado a una fibra óptica para transmitirse a través de la antena óptica hacia el espacio libre y es capturado en la etapa de recepción por la antena óptica consistente de un telescopio tipo Schmidt-Cassegrain de 14 pulgadas de diámetro, marca Meade modelo LX200-ACF. El telescopio concentra la energía recolectada en el plano focal del mismo, donde se coloca una etapa de colimación para acoplar la señal óptica de espacio libre a una fibra óptica monomodo estándar de telecomunicaciones hacia el receptor digital coherente formado por un acoplador óptico (un híbrido óptico de 90 grados) Kylia COH-28. Ahí se

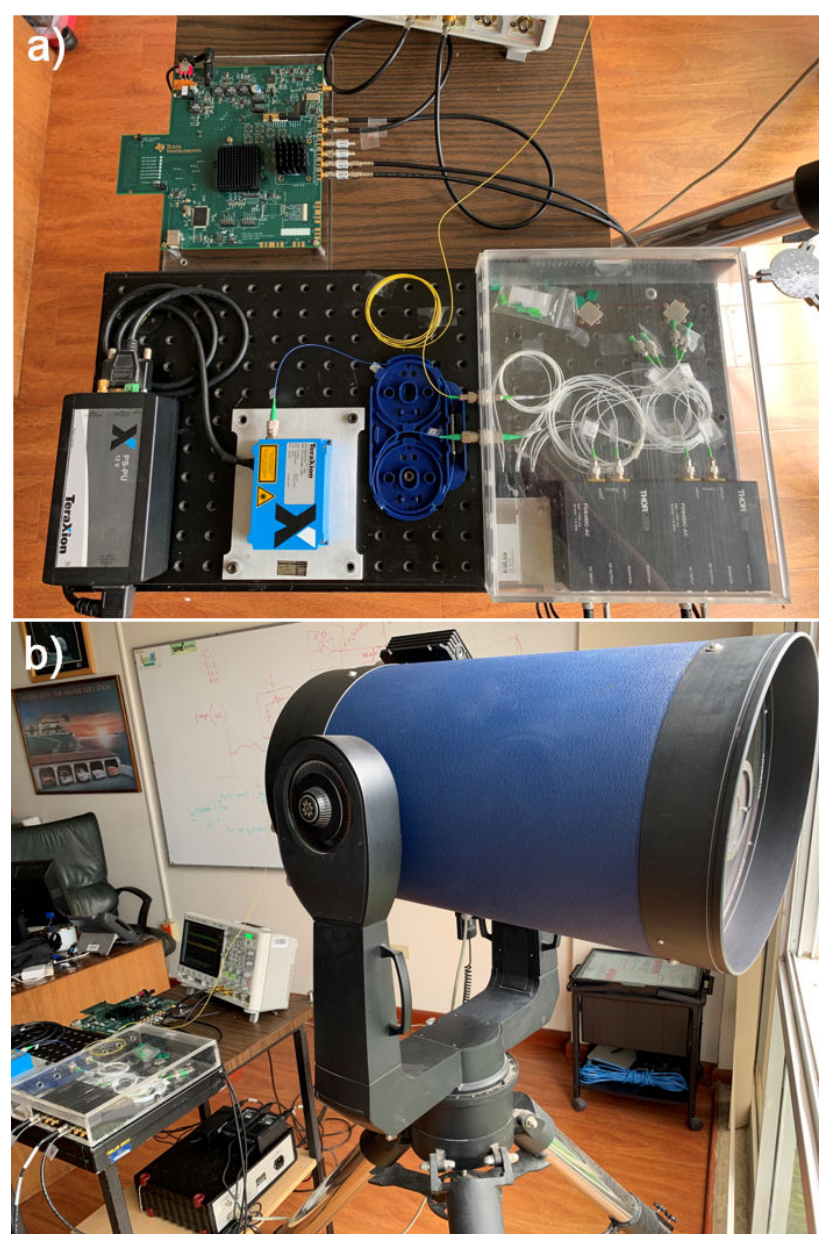

FIGURA 8. a) Receptor digital coherente para detección intradina de señales BPSK implementado. b) Antena óptica empleada con acoplamiento de fibra óptica hacia el receptor digital coherente.

mezcla con el oscilador local óptico, un láser Teraxion Purespectrum NLL con longitud de onda $1550 \mathrm{~nm}$ (más una diferencia de longitud de onda equivalente a $30 \mathrm{MHz}$ ) operando en modo free-running. El láser tiene un control de longitud de onda por compensación de temperatura manejado a través de software. La función del híbrido óptico es mezclar las señal de información con el oscilador local óptico, para extraer los componentes complejos de la señal de transmisión. Estas salidas ópticas son fotodetectadas por dos etapas de fotodetección balanceada Thorlabs PDB-480C-AC con un ancho de banda de $1.6 \mathrm{GHz}$. Así se obtienen obteniendo así los observables eléctricos (real e imaginario) que serán digitalizados por la tarjeta ADC12D1800RFRB/NOPB, donde son almacenados para su posterior procesamiento en el dominio del procesamiento digital de señales.

Dado que la tarjeta digitalizadora ADC12D1800RFRB /NOPB tiene un ancho de banda analógico de $1 \mathrm{GHz}$, encontramos experimentalmente que un buen compromiso entre la resolución de dicha tarjeta y la estabilidad de las fuentes ópticas conduce a una frecuencia intermedia (diferencia entre las frecuencias ópticas del oscilador local óptico y el campo óptico modulado) de alrededor de $30 \mathrm{MHz}$ para una velocidad de bit de $B_{r}=50 \mathrm{Mbps}$ (señal no retorno a cero, NRZ). En este 


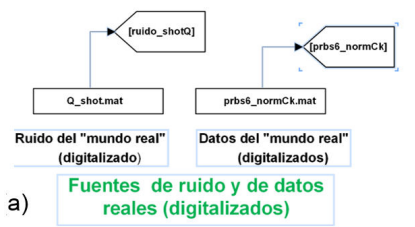

b)
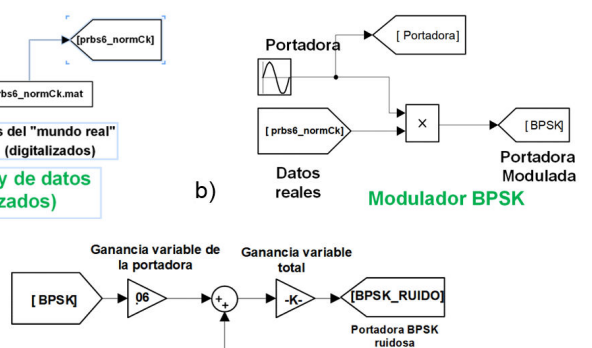

Control de

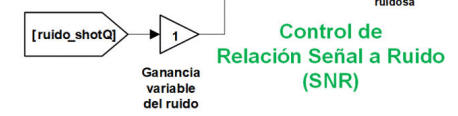

Ganancla
variable
del ruldo (SNR)

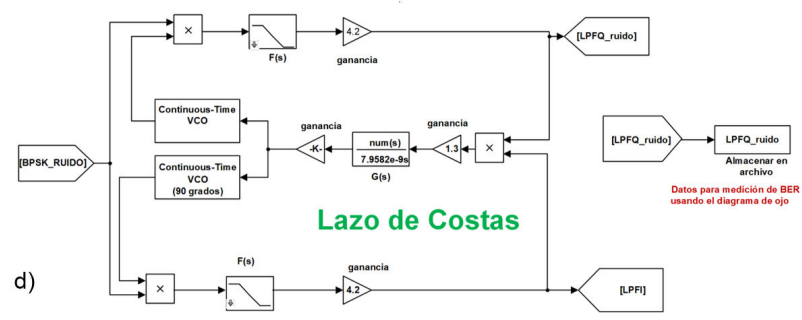

FIGURA 9. Bloques implementados para las simulaciones del lazo de Costas convencional y el intradino: a) Lectura de datos y ruido aditivo digitalizados, b) modulador de fase binaria (BPSK), c) control de nivel relación a ruido (SNR) para las simulaciones, d) Lazo de Costas.

este caso, la frecuencia intermedia corresponde a la portadora en el dominio eléctrico, lo que representa un caso "atípico", donde la velocidad del bit $\left(B_{r}=50 \mathrm{Mbps}\right)$ es mayor que la frecuencia de portadora $\left(f_{c}=30 \mathrm{MHz}\right)$, es decir, se tendrá una detección intradina [2].

Con el uso de la tarjeta digitalizadora ADC12D1800RF RB/NOPB adquirimos señales del mundo real para ser utilizadas en nuestras simulaciones. En particular, digitalizamos la señal de datos y la señal de ruido aditivo entregado a la salida de las etapas de fotorrecepción (empleando una frecuencia de muestreo de $4 \mathrm{GHz}$ ). Además, obtenemos la señal post-fotedetección digitalizada. Esta señal corresponde a una señal de detección intradina con modulación BPSK y es precisamente para demodular esta señal que se diseña el lazo de Costas intradino motivo de este escrito. Las simulaciones de los lazos de Costas las realizamos mediante el programa Simulink de Matlab y serán descritas a continuación. El objetivo es aprovechar la facilidad de Simulink para realizar la simulación mediante un diagrama a bloques que represente nuestro lazo diseñado. Además, Simulink tiene la opción de generar el código necesario para ser cargado en un dispositivo de procesamiento digital de señales (DSP), pues si bien estas simulaciones no operan en tiempo real sino más bien sirven para procesamiento fuera de línea ("off-line"), una vez validado el esquema demodulador se podría programar un dispositivo DSP con dicho código e integrarlo al sistema RDC para realizar procesamiento en tiempo real. Así, con base en los parámetros enlistados en las Tablas I y II, implementamos las simulaciones del lazo de Costas convencional y el intradino, respectivamente. La Fig. 9 muestra los diferentes
TABLA I. Parámetros para la implementación del lazo de Costas "convencional".

\begin{tabular}{cl}
\hline $\begin{array}{c}\text { Ganancias del VCO y } \\
\text { del comparador de fase } \\
\text { respectivamente }\end{array}$ & $\begin{array}{l}K_{0}=125.66 \times 10^{6} \mathrm{rad} / \mathrm{s} / \mathrm{V} \\
\text { es decir, } 20 \mathrm{MHz} / \mathrm{V} \text { y } \\
K_{d}=1 \mathrm{Volt} / \mathrm{rad}\end{array}$ \\
\hline Frecuencia de la portadora & $f_{c}=200 \mathrm{MHz}$ \\
\hline $\begin{array}{c}\text { Constante de tiempo } \\
\text { del filtro F(s) }\end{array}$ & $\tau=1.5915 \times 10^{-9} \mathrm{~s}$ \\
\hline Constantes de tiempo & $\tau_{1}=7.9582 \times 10^{-9} \mathrm{~s}$ \\
del filtro G(s) & $\tau_{2}=7.9577 \times 10^{-9} \mathrm{~s}$ \\
\hline Velocidad de Bit $\left(B_{r}\right)$ & $50 \mathrm{Mbps}$ \\
\hline
\end{tabular}

TABLA II. Parámetros para la implementación del lazo de Costas Intradino.

\begin{tabular}{cl}
\hline $\begin{array}{c}\text { Ganancias del VCO y } \\
\text { del comparador de fase } \\
\text { respectivamente }\end{array}$ & $\begin{array}{l}K_{0}=2 \pi \times 10^{6} \mathrm{rad} / \mathrm{s} / \mathrm{V} \\
\text { es decir, } 10 \mathrm{MHz} / \mathrm{V} \text { y } \\
K_{d}=1 \mathrm{Volt} / \mathrm{rad}\end{array}$ \\
\hline Frecuencia de la portadora & $f_{c}=30 \mathrm{MHz}$ \\
\hline Constante de tiempo & \\
del filtro F(s) & $\tau=6.2832 \times 10^{-6} \mathrm{~s}$ \\
\hline Constantes de tiempo & $\tau_{1}=1.7683 \times 10^{-7} \mathrm{~s}$ \\
del filtro G(s) & $\tau_{2}=5.3052 \times 10^{-8} \mathrm{~s}$ \\
\hline Velocidad de Bit $\left(B_{r}\right)$ & $50 \mathrm{Mbps}$ \\
\hline
\end{tabular}

bloques empleados. Es pertinente mencionar que ambas simulaciones emplean los mismos bloques pero con diferentes parámetros, lo cual determina el comportamiento dinámico de cada uno de ellos.

Los elementos empleados en la simulación mostrados en la Fig. 9 contienen una leyenda que indica el significado de cada uno de ellos; por ejemplo, en la Fig. 9a), la leyenda correspondiente a Q_shot.mat se relaciona con un archivo de datos (por eso, la extensión .mat) que es la señal de Ruido del mundo real "digitalizado" (adquirida mediante digitalización). Existen también los bloques denominados "Goto" y "From" que se utilizan por claridad para evitar trazar líneas que conecten los diversos componentes de la simulación. Si la flecha "entra" al bloque, se trata de un bloque indicando que direcciona una señal hacia un punto en correspondencia con la etiqueta. Así, en la Fig. 9a) la salida del bloque rectangular "Q_shot.mat", entra (de acuerdo a la dirección de la punta de la flecha) al bloque "Goto" que direcciona dicha señal hacia el punto que tenga un bloque "From" con la etiqueta "ruido_shotQ" (ver Fig. 9c). En este caso, sabemos que es un bloque "From" pues la señal "sale" del mismo (de acuerdo a la dirección de la punta de la flecha) hacia un amplificador con la etiqueta "Ganancia variable del ruido". De esta forma podemos conectar la salida del bloque Q_shot.mat con la entrada del amplificador mencionado sin requerir trazar líneas para unirlos. El resto de los bloques tienen correspondencia con el diagrama de la Fig. 5. 


\begin{tabular}{ccccc}
\hline \hline TABLA III. Tasa de bit erróneo (BER) para diferentes valores de relación señal a ruido (SNR). & \\
\hline $\begin{array}{c}\text { Relación } \\
\text { señal a ruido } \\
\text { (SNR) en dB }\end{array}$ & Valor teórico del & Valor del BER & Valor del BER & Valor del BER \\
& BER para el & para el lazo de Costas & para el lazo de Costas & para el lazo de Costas \\
lazo de Costas & convencional obtenido & intradino obtenido & intradino obtenido \\
convencional & mediante simulación & mediante simulación & de datos experimentales \\
\hline 7.32 & $1 \times 10^{-2}$ & $1.01067 \times 10^{-2}$ & $2.26528 \times 10^{-2}$ & $4.9985 \times 10^{-2}$ \\
9.78 & $1 \times 10^{-3}$ & $1.02685 \times 10^{-3}$ & $4.67832 \times 10^{-3}$ & $1.5003 \times 10^{-2}$ \\
11.4 & $1 \times 10^{-4}$ & $1.00269 \times 10^{-4}$ & $6.02839 \times 10^{-4}$ & $2.2924 \times 10^{-3}$ \\
12.57 & $1 \times 10^{-5}$ & $1.06135 \times 10^{-5}$ & $5.28218 \times 10^{-5}$ & $3.5842 \times 10^{-4}$ \\
13.5 & $1 \times 10^{-6}$ & $1.08958 \times 10^{-6}$ & $2.43088 \times 10^{-6}$ & $2.1424 \times 10^{-5}$ \\
\hline
\end{tabular}

\subsection{Evaluación de desempeño}

La medida más importante del buen funcionamiento de un sistema de comunicaciones digitales es la tasa de bit erróneo (BER, por sus siglas en inglés, Bit error rate) en función de la relación señal a ruido (SNR, por sus siglas en inglés, signalto-noise ratio) en la etapa de recepción y de demodulación. Así, hemos realizado simulaciones, para varios valores típicos de BER vs SNR, en primer lugar, para el lazo Costas convencional y posteriormente para el intradino. Además, hemos obtenido el BER para el lazo de Costas intradino utilizado como demodulador del observable eléctrico post-fotedetección digitalizado. A este último le llamaremos "lazo de Costas intradino experimental". La medición de la tasa de bit erróneo se puede llevar a cabo con varias técnicas, por ejemplo, comparando los bits transmitidos con los recibidos uno a uno. Esto para nuestro caso no es muy eficiente pues implica realizar un proceso denominado regeneración digital y enfasamiento de los datos demodulados y regenerados con los transmitidos. Este proceso requiere el uso de una gran cantidad de recursos tanto de tiempo como de memoria de computadora para cada valor de relación señal a ruido. Una técnica alternativa, menos demandante pero ampliamente usada en los sistemas de comunicaciones ópticas digitales, es el denominado diagrama de ojo [23-25], el cual decidimos emplear con un algoritmo que implementamos en Matlab. En la Fig. 10 se muestra un ejemplo de los diagramas de ojo obtenidos, mientras que en la Fig. 11 se muestran algunas señales típicas de las simulaciones en el dominio del tiempo. Los resultados tanto de nuestras simulaciones como del "lazo de Costas intradino experimental" se muestran en la Tabla III y la Fig. 12, donde se observa que el desempeño del lazo de Costas convencional simulado es muy cercano al valor teórico mientras que el desempeño del Costas intradino simulado, como era de esperarse, es inferior debido a la presencia de términos de portadora en el ancho de banda de la información, lo cual provoca un deterioro en la relación señal a ruido que a su vez afecta en la tasa de bit erróneo (incrementándola).

Si bien el desempeño del "lazo de Costas intradino experimental" es cercano al lazo de Costas intradino simulado, existe una discrepancia para todos los valores de relación señal a ruido (SNR). Desde nuestro punto de vista, esta dife- rencia puede atribuirse a la existencia del ruido de fase de las fuentes ópticas utilizadas. Por sencillez, el ruido de fase no fue incluido en nuestras simulaciones. Existen, modelos para dicho ruido que pueden utilizarse en lazos de Costas ópticos tanto para el caso homodino como para el heterodino [26]; sin embargo, a lo mejor de nuestro conocimiento, no existe dicho modelo para lazos de Costas intradinos. Actualmente trabajamos en la obtención del modelo de dicho ruido para ser incluido en un próximo manuscrito. Sin embargo, como también puede observarse en la gráfica, el lazo de Costas intradino sí funciona como demodulador de señales BPSK con valores de BER aceptables, no tan alejados del valor teórico. Creemos que la diferencia del lazo de Costas intradino simulado con respecto al valor teórico puede deberse al hecho de que las ecuaciones empleadas para el diseño del lazo de Costas intradino fueron las mismas que se usan para un lazo de Costas convencional sin tomar en cuenta el deterioro de la relación señal a ruido mencionado anteriormente.

Dado que el objetivo inicial de este trabajo fue mostrar la factibilidad de emplear el lazo de Costas para demodulación de señales BPSK cuando la frecuencia de portadora es menor que la velocidad de los datos, consideramos que se ha cumplido con el objetivo. Sin embargo, es conveniente poder predecir el valor esperado de la tasa de bit erróneo para una relación señal a ruido y un valor de ruido de fase dados. Por este motivo, actualmente trabajamos en el análisis y desarrollo de las ecuaciones pertinentes y nuestros resultados serán motivo de un trabajo posterior.

\section{Conclusiones}

A lo mejor de nuestro conocimiento, en este trabajo hemos presentado por primera vez un lazo de Costas en el dominio del procesamiento digital de señales empleado para la demodulación de un campo óptico con modulación BPSK perfecta en un esquema de detección intradino. Para el diseño de dicho lazo nos apoyamos en la teoría desarrollada para lazos de Costas convencionales, donde la frecuencia de portadora es mayor que la velocidad del bit. Dado que el lazo de Costas desarrollado tiene por objetivo ser empleado en un esquema real para comunicaciones ópticas con modulación BPSK, 
hemos presentado también el esquema práctico implementado de transmisión y recepción digital coherente intradina. Es pertinente mencionar que si bien las simulaciones equivalen a un procesamiento fuera de línea, con la generación del código pertinente a partir de dichas simulaciones podría programarse un dispositivo de procesamiento digital de señales para ser integrado en un esquema de recepción digital coherente intradino y así el lazo de Costas operaría en tiempo real. Desde nuestro punto de vista, el desempeño del lazo de Costas intra- dino evaluado mediante diagramas de ojo es suficientemente satisfactorio como prueba de concepto. Sin embargo, su operación requiere ser optimizada, para lo cual resulta necesario poder calcular la tasa de bit erróneo teórica en función tanto de la relación señal a ruido como del ruido de fase de las fuentes ópticas. Actualmente trabajamos en la deducción de las fórmulas pertinentes y los resultados obtenidos serán objeto de una próxima publicación.
1. R. Hui, Introduction to Fiber-Optic Communications, 1 st ed. (Academic Press, San Diego, CA, 2020) pp. 337-339,417-418, 488-489,555556. https://www.elsevier.com/books/ introduction-to-fiber-optic-communications/ hui/978-0-12-805345-4

2. M. Nakazawa, K. Kikuchi, y T. Miyazaki (Eds.), High Spectral Density Optical Communication Technologies, (Springer-Verlag, Berlin Heidelberg, 2010), pp. 11-20. https://link.springer.com/book/10.1007/ 978-3-642-10419-0

3. L. N. Binh, Advanced Digital Optical Communications, (CRC Press Taylor \& Francis Group, Boca Raton, FL, 2015), pp. 1-11. https://www.routledge.com/ Advanced-Digital-Optical-Communications/ Binh/p/book/9781138749542

4. Z. Zhang et al., High-speed Coherent Optical Communication with Isolator-free Heterogeneous $\mathrm{Si} / \mathrm{III}-\mathrm{V}$ Lasers, Journ. of Lightw. Techn. (August), 2020, 1, https://doi:10. $1109 /$ JLT.2020.3015738

5. M. Xu et al., High-performance coherent optical modulators based on thin-film lithium niobate platform, Nat Commun 11 (2020) 1. https://doi.org/10.1038/ S41467-020-17806-0

6. J. Wu and X. Ke, Experimental on correction of wavefront distortion in coherent optical communication system, Proc. SPIE Sixth Symposium on Novel Optoelectronic Detection Technology and Applications 11455 (2020) 114552R. https: //doi.org/10.1117/12.2563985

7. S. Ritter, An Optimum Phase Reference Detector for Fully Modulated Phase Shift Keyed Signals, IEEE Trans. on Aero. and Elect. Syst. AES-5 (1969) 627. https://doi.org/10. 1109/TAES.1969.309945

8. J. G. Proakis, Digital Communications 4th Edition, (McGrawHill, New York, 2001), pp. 339-356.

9. C.Y. Mei, A.Z. Sha'ameri, y B. Boashash, Efficient phase estimation for the classification of digitally phase modulated signals using the cross-WVD: a performance evaluation and comparison with the S-transform, EURASIP J. Adv. Signal Process. 65 (2012) 1. https://doi.org/10.1186/ 1687-6180-2012-65

10. J. P. Costas, Synchronous Communications, Proc. of the IRE, 44 (1956) 1713. https: //doi.org/10.1109/JRPROC. 1956.275063
11. J. A. Lopez Leyva, A. Arvizu Mondragon, E. Garcia, F. J. Mendieta, E. Alvarez Guzman, P. Gallion, Detection of phase-diffused weak-coherent-states using an optical Costas loop, Opt. Eng. 51 (2012) 105002, https: // doi .org/10. $1117 / 1.0 \mathrm{E} .51 .10 .105002$

12. Q. Xu, A. Arvizu Mondragon, P. Gallion and F. J. Mendieta, Homodyne In-Phase and Quadrature Detection of Weak Coherent States With Carrier Phase Tracking, IEEE Journ.of Sel. Top. in Quant. Elect. 15 (2009) 1581.https://doi.org/ $10.1109 /$ JSTQE.2009.2023803

13. A. Arvizu-Mondragón, et al., Optical Communication Receiver Based on a Switched-Quadrature Costas Loop, Journ. of App. Res. and Techn. 9 (2011) 443. https://doi.org/ $10.22201 /$ icat.16656423.2011.9.03.436

14. J. Spilker, Digital Communications by satellite, (Prentice-Hall, Englewood Cliffs, NJ 1977) pp. 297.

15. F. M. Gardner, Phaselock techniques, 3rd edition. (John Wiley \& Sons, Palo Alto, California, 2005), pp.2-14, 97121. https://www.wiley.com/en-us/Phaselock+ Techniques $\div 2 \mathrm{C}+3 \mathrm{rd}+$ Edition-p-9780471732686

16. A. Blanchard, Phase locked loops, application to coherent receiver design, (John Wiley and sons, New York, NY 1976) pp. 3-7, 81-138, 302-314.

17. D. R. Stephens, Phase-Locked Loops for Wireless Communications: Digital, Analog and Optical Implementations, 2nd edition, (Kluwer Academic Publishers, Norwell, Massachusetts 2002) pp. 1-56. https : //www . springer. com/gp/ book/9780792376026

18. F. M. Gardner, Phaselock techniques, 2nd Edition, (John Wiley and Sons, New York, NY 1979) pp.8-11, 21-31, 43-90.

19. M. Schwartz, Transmisión de información, modulación y ruido, tercera edición, (McGraw-Hill, México 1983) pp. 233.

20. J. Feigin, Practical Costas loop design, RF design, (January 2002) pp. 20.

21. M. Simon, W. Lindsey, Optimum Performance of Suppressed Carrier Receivers with Costas Loop Tracking, IEEE Trans. on Comm. 25 (1977) 215. https: / / doi .org/10.1109/ TCOM.1977.1093805

22. J. M. Steber, PSK Demodulation (Part 1), Watkins-Johnson Company Tech-notes 11 (1984) 1. 
23. G. Breed, Analyzing Signals Using the Eye Diagram, High Frequency Electronics, (November 2005), pp.50. https://www.highfrequencyelectronics.com/ Nov05/HFE1105_Tutorial.pdf

24. Agilent Technologies, 71501 Eye-Diagram Analysis User's Guide, pp1. http://literature.cdn.keysight. com/litweb/pdf/70874-90023.pdf
25. Anritsu Corporation, Understanding Eye Pattern Measurements, (Anritsu Application Note, 2010), pp. 1.

26. A. Arvizu-Mondragón, F.J. Mendieta-Jiménez, J. de D. Sánchez-López, Estimadores de fase óptica en sistemas modernos de comunicaciones. Homodinas Ing. invest. y tecnol., 10 (2009) 187. http://dx.doi.org/10.22201/fi. 25940732e.2009.10n3.017 